\title{
BMJ Open Effects of a peer support programme for youth social services employees experiencing potentially traumatic events: a protocol for a prospective cohort study
}

\author{
Stephane Guay, ${ }^{1,2}$ Nicole Tremblay, ${ }^{2}$ Jane Goncalves, ${ }^{2}$ Henriette Bilodeau, ${ }^{3}$ \\ Steve Geoffrion ${ }^{2,4}$
}

To cite: Guay S, Tremblay N, Goncalves J, et al. Effects of a peer support programme for youth social services employees experiencing potentially traumatic events: a protocol for a prospective cohort study. BMJ Open 2017;7:e014405. doi:10.1136/ bmjopen-2016-014405

- Prepublication history and additional material are available. To view please visit the journal (http://dx.doi.org/ 10.1136/ bmjopen-2016-014405)

Received 22 September 2016 Revised 13 February 2017 Accepted 24 March 2017

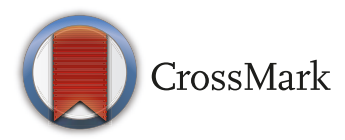

'École de criminologie, Université de Montréal, Montreal, Quebec, Canada ${ }^{2}$ Trauma Studies Centre, Institut universitaire en santé mentale de Montréal, Montreal, Quebec, Canada

${ }^{3}$ Département d'organisation et ressources humaines, Université du Québec à Montréal, Montreal, Quebec, Canada

¿École de psychoéducation, Université de Montréal, Montreal, Quebec, Canada

Correspondence to Dr. Stephane Guay; stephane. guay@umontreal.ca

\section{ABSTRACT}

Introduction The use of peer support programmes to help workers experiencing potentially traumatic events (PTE) has increased in high-risk organisations in the last decades. However, the scientific evidence of its effectiveness is still very limited. This paper aims to describe the protocol of a prospective cohort study that assesses the efficacy of a peer support programme among youth social services employees exposed to a PTE at work on psychological well-being, work functioning and needs of support.

Methods and analysis This is a mixed-methods prospective study that will examine workers' evolution four times over a 12-month period in Canada. This study involves: (1) quantitative data obtained through selfadministrated questionnaires among 222 workers, and (2) qualitative in-depth interviews with a subsample of 45 workers. This study will compare findings from a cohort who received the support of a peer following a PTE (peer support-experimental protocol) as part of the experimental protocol of the Montreal Youth Social Services-University Institute (MYSS-UI), the second group of workers did not ask for the peer support (no peer support-experimental protocol) but was part of MYSS-UI, and the third group received standard organisational support from the Monteregie Youth Social Services (MYSS) (standard organisational protocol).

Ethics and dissemination The protocol and informed consent form complied with the ethics guidelines of the MYSS-UI. The Research Ethics Board of MYSS-UI and MYSS reviewed and accepted the protocol as required. The results of the study will be published in peerreviewed journals, presented at research and general public conferences, disseminated via a public report for the institute that funded the project and for all workers. Results of this study will influence decision making regarding intervention policies following PTE and peer support interventions may be expanded throughout the youth social services in Canada and worldwide.

\section{INTRODUCTION}

Post-traumatic stress disorder (PTSD) is one of the most frequent mental illnesses
Strengths and limitations of this study

- This study is designed to address the lack of empirical data supporting the effectiveness of peer support for high-risk organisations.

- This study will assess the psychological distress, work functioning and needs regarding support of three cohorts of workers who have initially experienced a potentially traumatic event (PTE) four times during 12 months.

- This study uses a mixed-methods design which combines quantitative and qualitative research methods. As a result of a process of triangulation, we will give a comprehensive view on the efficacy of interventions under study.

- Due to the nature of the study, we cannot control the assignment.

- In spite of youth social services' efforts and our own, to raise awareness about PTEs, some workers may not recognise all types of PTEs as such. Increased awareness among workers through this research project, however, could result in positive change.

associated with exposure to a traumatic event. For example, according to a systematic review on the consequences of workplace violence, $5 \%-32 \%$ of victimised workers met diagnostic criteria for PTSD. ${ }^{2}$ The distinguishing feature of PTSD when compared with other disorders-or states such as psychological distress-is the necessity of direct or indirect exposure to a potentially traumatic event (PTE) (criterion A). According to the DSM-5 , 3 , an event is considered to be traumatic when it involves a perceived threat to life or physical integrity (ie, assault, rape) or witnessing such acts (ie, witnessing a murder, seeing dead bodies). PTEs are a serious issue, potentially leading to many functional impairments, whether they be psychological or occupational in nature. ${ }^{3}$ For example, commonly 
reported outcomes of workplace violence include stress, burnout, fear, psychological distress, lowered quality of life and diminished job satisfaction. Furthermore, these consequences often lead to higher rates of intentions to quit, actual turnover and absenteeism. ${ }^{4-8}$

Some organisations are 'at-risk' for PTEs and therefore staff with PTSD symptomology. Numerous of these organisations have developed programmes for staff to help them cope with the consequences of PTEs. ${ }^{9}$ Some initiatives, such as Critical Incident Stress Management, ${ }^{10}$ have the distinction of being offered by peers specifically trained to work with their colleagues. According to the first international peer support guidelines, ${ }^{9}$ the main goals of peer support are 'to provide an empathic, listening ear; to provide low-level psychological intervention; to identify peers who may be at risk to themselves or others; and to facilitate pathways to professional help' ( $p$. 137). The peers, who have a more detailed knowledge of the organisation and its members, can foster trust with those affected and increase the effect of cohesion and support within the group. ${ }^{11}$

So far, research on peer support programmes is very scarce and mainly descriptive. Studies are generally conducted among small samples, and among cross-sectional or longitudinal designs without comparison groups. ${ }^{9}$ Thus, few of them have been validated and their efficacy remains to be investigated. To their defence, implementing a randomised trial is complex due to the nature of the support, especially because randomisation would lead to the exclusion of some victims in the programme. $^{12}$

In psychiatric hospital settings, programmes involving peer helpers were designed to help prevent PTSD symptoms among employees of psychiatric hospitals. ${ }^{13-15}$ The Assaulted Staff Action Program (ASAP) is a peer-help crisis intervention programme that aims to address the psychological needs of employees who are victims of patient assaults. It operates at a system-wide level and on a voluntary basis. When an assault occurs, an ASAP team member ensures that any safety or physical health concerns have been addressed, gathers information regarding the assault and introduces the victims to ASAP services. Upon approval from the assaulted employee, the ASAP team member conducts an assessment for any symptom of psychological trauma and offers assistance with regard to work-related tasks, providing a support network, and helping the employee understand the reasons for the assault.

Some of the evaluations show that these programmes are popular with employees ${ }^{1516}$ and managers ${ }^{17}$ and they can help educate employees on post-traumatic stress and encourage help-seeking behaviours following a PTE. ${ }^{18}$ The ASAP is one of the most frequently assessed PTSD preventive programmes involving peer support in healthcare. $^{13-15}$ The authors observed an association between programme participation and a reduction in post-traumatic symptoms. However, the methodology used (ie, no control group) did not allow for a conclusion about its efficacy to prevent or alleviate post-traumatic sequelae. ${ }^{13}$ Moreover, cognitive behavioural therapy (CBT), which appears in a systematic literature review ${ }^{19}$ to be the most promising early intervention for victims of violent crime, is not part of the ASAP approach.

In sum, one of the main issues confronting the field of peer support for high-risk organisations is the relative lack of empirical data supporting its effectiveness. ${ }^{9}$ This study is designed to address these limits in comparing three cohorts of workers at high risk of experiencing PTE, namely child protection workers. The aim of the study is to compare in a prospective design the psychological wellbeing, work functioning and needs regarding support of three cohorts of workers during 12 months who have initially experienced a PTE. More precisely, we will study the impact of structured peer support and standard organisational support following a PTE on psychological distress, measured through levels of acute stress disorder, PTSD, perceived stress, depression and anxiety. We will also study the impact of these different sources of support on work functioning, measured through quality of life at work, presenteeism, absenteeism, as well as perceptions of workplace security.

The first group received the support of a peer following a PTE (peer support-experimental protocol) as part of the experimental protocol of the youth social services, the second group of workers did not ask for the peer support (no peer support-experimental protocol) but was part of the same Youth Social Services, and the third group received standard organisational support from another youth social services (standard organisational cohort), which does not include a peer support intervention.

\section{Child protection workers: an at-risk profession}

Child protection workers face special dangers on the job. ${ }^{4} 62021$ These employees generally directly work in contact with vulnerable youth, who experience a disproportionate risk of mental and behavioural health problems and developmental disorders compared with their counterparts outside youth protection settings. ${ }^{22} 23$ In this sector of activity, workplace violence is one of the most common forms of PTEs. Violence and aggression at work are defined as 'a range of behaviours or actions that can result in harm, hurt or injury to another person, regardless of whether the violence or aggression is physically or verbally expressed, physical harm is sustained or the intention is clear. ${ }^{24}$ Prevalence rates of workplace violence towards these employees vary widely in the literature, notably because of methodological differences among studies. In a survey conducted among 586 educators in youth centres in the Province of Quebec, Canada, Geoffrion and Ouellet ${ }^{25}$ found that $53.9 \%$ had been victims of physical assault by a youth in the previous year. Sixty per cent had been assaulted more than once and $10 \%$ more than five times during the year. In addition, $95 \%$ of the workers had witnessed at least one incident of violence. 
Recent data collected from 310 participants in one of the two organisations under study in the present protocol (Monteregie Youth Social Services; MYSS) indicate that these workers are occasionally exposed to self-destructive behaviours (eg, self-harm gesture, suicide attempts, suicide). ${ }^{26}$ Also, exposure of social workers to indirect violence related to distress manifested by the youth is associated with post-traumatic stress reactions, such as intrusive thoughts, sleep and concentration difficulties. ${ }^{27}$ Indeed, some child protection workers also develop PTSD following PTE exposure, ${ }^{28-30}$ which is characterised by intrusion symptoms (eg, flashbacks, distressing dreams), persistent avoidance (eg, of feelings, thoughts); negative alterations in cognitions and mood (eg, persistent negative emotional state); and marked alterations in arousal and reactivity (eg, irritable behaviour, hypervigilance). ${ }^{3}$ Rhee $e t a l^{30}$ analysed the data obtained from 255 full-time child protection workers from 43 child protective agencies in South Korea. They found that the average intensity of post-traumatic stress symptoms as measured by the Impact of Event Scale (IES-R-K) was as high as 28.73 ( $\mathrm{SD}=20.05$ ), that is to say, higher than the cut-off score for PTSD selection which is 24. These data also highlight that $80 \%$ of individuals diagnosed with PTSD struggle with at least one comorbidity, including depression, anxiety or substance use disorder. Given the occurrence and numerous negative effects of PTE and PTSD, researchers and organisations have become gradually more interested in defining strategies contributing to the caring and healing of workers affected by consequences of traumatic events.

\section{A peer support programme designed for youth social services employees}

In 1999, the Montreal Youth Social Services in the Province of Quebec, Canada (MYSS-UI; Montreal Youth Centre-University Institute) designed, and revised in 2005 , an intervention including a post-traumatic specialised peer support protocol for workers exposed to PTE. The MYSS-UI associated management measures to prevent and mitigate the development of post-traumatic reactions, acute stress and PTSD among workers who experienced PTE. The main characteristics of the programme are presented in table 1 and the whole programme has been described elsewhere. ${ }^{31}$ To sum up, the protocol indicates the sequence of actions to be taken and specifies the division of responsibilities between all involved and concerned individuals. A post-traumatic intervention team (PTIT), composed of stakeholders (peers) of the MYSS-UI, with over 10 years of clinical experience and specifically trained in post-traumatic counselling, is responsible for the clinical aspects of the intervention. The protocol favours individual meetings on a voluntary basis, with those most directly affected by the event. Through specific interventions, meetings with a peer helper primarily aim to promote the integration of the event and the adaptation of the affected person as well as to detect other workers at risk for developing PTSD. The postimmediate intervention conducted by the peer support worker is based on a model of brief early CBT to individuals with acute stress disorder in order to prevent or mitigate the development of post-traumatic reactions. ${ }^{32}$ The peer helpers are trained to key cognitive and behavioural intervention strategies of this model, apart from the use of in vivo exposure technique that requires more specialised training (box 1 ).

A first evaluative phase of the programme was used to analyse the theoretical and empirical relevance of this intervention and measure the extent of implementation within the organisation. ${ }^{31}$ The results indicated that the workers are all well informed about the existence of the peer support programme. However, although the programme may be well known, it is not systematically used. Questions regarding the reasons why they do not use the support offered, and more generally about their needs, have to be addressed. ${ }^{31}$ The MYSS-UI peer support programme is unique in the province of Quebec, as all the other youth social services centres use a standard procedure based on professional help, as it is the case of the MYSS.

More precisely, in the MYSS, a guide regarding prevention and management during outbreaks of violence by clients directed to staff specifies steps to be followed and determines roles and responsibilities. The MYSS provides clinical, administrative and judicial interventions, in which the immediate superior and the Health and Safety Office play a central role. However, none of the immediate superiors are trained to post-traumatic CBT prevention interventions. Although many of these measures are similar to those of the PTIT of the MYSS-UI, the two protocols differ primarily in terms of the clinical services available to affected workers, namely due to the optional recourse to peer help support counsellors trained in CBT (see table 1).

\section{OBJECTIVES}

The aim of the study is to assess among youth social services workers affected by PTE whether (1) psychological distress and (2) work functioning improve through the PTIT peer support protocol among youth social services workers who experienced a PTE. This study also aims to: (3) explore the motives for using or not using the PTIT's services as offered by the MYSS-UI, (4) explore the support needs of workers exposed to PTEs and working with the MYSS-UI or MYSS in the year following the event, and (5) explore the perceptions of different stakeholders regarding the PTIT. The findings will inform both design of interventions and the design of research for evaluating such interventions. Findings will influence decision making and lead to interventions tailored to the needs of workers. Findings may also support implementation of similar interventions for child protection organisations in Canada and worldwide.

\section{METHOD}

Participants and procedures

The study includes potentially all employees of the MYSS-UI and MYSS, which represent 2699 and 1752 


\begin{tabular}{|c|c|c|c|}
\hline Phase & Intervention & MYSS & MYSS-IU \\
\hline \multirow[t]{4}{*}{$\begin{array}{l}\text { Immediate phase } \\
(0-4 \text { hours })\end{array}$} & $\begin{array}{l}\text { Physical security of victims and first aid by the staff } \\
\text { present }\end{array}$ & $\checkmark$ & $\checkmark$ \\
\hline & Psychological first aid & $\begin{array}{l}\text { By the staff present } \\
\text { and by the IS }\end{array}$ & By the staff present \\
\hline & Session of individual or group verbalisation & $\checkmark$ & \\
\hline & Inform the hierarchy & $\checkmark$ & $\begin{array}{l}\text { Evaluation of the need for } \\
\text { a coordinating committee } \\
\text { or postvention by the } \\
\text { management }\end{array}$ \\
\hline \multirow[t]{8}{*}{$\begin{array}{l}\text { Postimmediate phase } \\
\text { (4-72 hours) }\end{array}$} & $\begin{array}{l}\text { Assessment of the state and needs of the victims } \\
\text { and witnesses by the IS }\end{array}$ & $\checkmark$ & $\checkmark$ \\
\hline & Inform the HSO & $\begin{array}{l}\text { And of all at-risk } \\
\text { workers by the IS }\end{array}$ & By the IS \\
\hline & Offer different external options of support & $\checkmark$ & $\checkmark$ \\
\hline & Offer internal support (peer support) & & $\begin{array}{l}\text { For the peer support cohor } \\
\text { only }\end{array}$ \\
\hline & $\begin{array}{l}\text { Solicit a specialist in postvention if necessary by the } \\
\text { HSO }\end{array}$ & $\checkmark$ & \\
\hline & Support in administrative and justice procedures & By the HSO & By the IS \\
\hline & Vigilance and re-evaluation of the needs if necessary & $\checkmark$ & $\checkmark$ \\
\hline & $\begin{array}{l}\text { Application of administrative measures if necessary } \\
\text { (two paid sick days) }\end{array}$ & & $\checkmark$ \\
\hline \multirow[t]{6}{*}{$\begin{array}{l}\text { Midterm phase } \\
\text { ( } 72 \text { hours and more) }\end{array}$} & $\begin{array}{l}\text { Use of an external assistance resource if necessary } \\
\text { (employee assistance programmes) }\end{array}$ & $\checkmark$ & $\checkmark$ \\
\hline & $\begin{array}{l}\text { Participation in individual meetings of victims } \\
\text { conducted by a peer support member }\end{array}$ & & $\begin{array}{l}\text { For the peer support cohor } \\
\text { only }\end{array}$ \\
\hline & $\begin{array}{l}\text { Coordination of maintenance and reintegration } \\
\text { strategies for employment by the HSO in } \\
\text { collaboration with the IS }\end{array}$ & $\checkmark$ & \\
\hline & $\begin{array}{l}\text { Vigilance, re-evaluation of the needs and offer } \\
\text { additional support by the IS }\end{array}$ & & $\checkmark$ \\
\hline & $\begin{array}{l}\text { Arrangement of the working conditions and support } \\
\text { to return to work if necessary by the IS }\end{array}$ & & $\checkmark$ \\
\hline & Postevent analysis & $\begin{array}{l}\text { By the IS and the } \\
\text { HSO }\end{array}$ & By the IS \\
\hline
\end{tabular}

HSO, Heath and Security Office; IS, immediate superior; MYSS, Monteregie Youth Social Services; MYSS-UI, Montreal Youth Social ServicesUniversity Institute.

employees, respectively. They work in different units including residential care (group care, secure care, juvenile detention) or in youth and family residencies. Thus, the study is open to all the employees regardless of job titles (eg, supervisors, educators, social workers, security agents, cleaning staff) who were exposed directly or indirectly to a PTE as part of their job within the last month. Potentially eligible traumatic events accepted are (a) being a victim or witness of a PTE or (b) learning that a youth was exposed to a physical aggression (eg, punch, kick, armed attack), verbal abuse (eg, threats, intimidation) or sexual abuse. Also eligible are witnessing a suicidal gesture (eg, suicide attempts), maltreatment or death (eg, suicide, accidental death).
Reporting the PTE to the employer or Health and Security Office is not mandatory for inclusion in the study.

\section{Recruitment}

The recruitment started in January 2016. Several methods are used to recruit participants. Informational emails on the study are sent at regular time intervals to all employees with an email address of the establishment. The study promotion is made via the intranet of the youth centres and by the first line manager and members of the Health and Security Office. In both facilities, posters are hung, union members promote the project among all workers, and the research team meets the management team on a regular basis. In the MYSS-UI, the study is also promoted 


\section{Box 1 Training objectives for peer members}

Peer support intervention members are trained to:

1. Know the definition and clinical features of post-traumatic stress.

2. Be able to identify potentially traumatic events.

3. Understand the professional, personal and family consequences of a potentially traumatic event.

4. Know the protective factors and factors predisposing, precipitating and maintaining post-traumatic stress disorder.

5. Understand the benefits of concerted preventive action in the ional support without support forganizationorganisation.

6. Know the history of defusing procedures and debriefings.

7. Know the immediate intervention measures following a traumatic event.

8. Know the immediate postintervention measures following a traumatic event.

9. Know the roles of all participants at the immediate and postimmediate interventions.

10. Be aware of the prevention of compassion fatigue among helpers themselves.

11. Understand the principles of cognitive-behavioralbehavioural intervention.

12. Master respiratory rehabilitation and progressive muscle relaxation.

13. Master the use of Beck Socratic questioning.

14. Master the use of Meichenbaum stress inoculation.

by the peer helper during the first telephone contact with an employee who uses the services after being exposed to a PTE.

Interested workers contact the research team or fill out a contact authorisation form allowing their immediate supervisor or peer helper to send their contact information to the research team via email or phone. Instructions on how to contact the research team are also included in all research-promoting communications made within the MYSS-UI and MYSS. Interested participants are called in the following days by the research coordinator. During the initial contact with the research team, interested workers receive information on the progress and objectives of the study to decide if they agree to participate. If they agree, the research team emails a consent form summarising the nature of the study and its expected benefits and inconveniences that participants must sign and return electronically or by mail.

\section{Quantitative data}

All the MYSS-UI and MYSS workers will be evaluated four times during the 12-month period following the event: during the first month and then at 2, 6 and 12 months after the event. The collection of quantitative data is performed using computerised questionnaires on the SurveyMonkey platform. Data are then downloaded in IBM SPSS V.21 software. When the signed consent form is returned, the research assistant assigns an identification (ID) number to each participant and provides, by email, the internet survey link with its ID number. The participating workers have the possibility to be released for an hour to complete the questionnaire during their shift. They also receive a $\$ 35$ compensation for each completed assessment. They have the option to complete the questionnaire from home or on their worksite. When the questionnaire is completed on their worksite, an isolated workstation is accessible to guarantee privacy. At each time point, a link to the internet survey will be sent to the respondents by email. A reminder email and phone call are done at each measurement time when necessary. The data are collected via self-administered paper questionnaire if participants do not want or cannot complete the questionnaire online.

\section{Qualitative data}

Qualitative data on participants' support needs following the event are collected by the way of semistructured interviews over the phone. During the first telephone contact with the research coordinator, the workers are invited to participate in these interviews in parallel with the completion of questionnaires (ie, four measurement times). This procedure will be done until the required number of participants is reached. We intend to recruit 60 participants with a final subsample of 15 participants per cohort (ie, taking into account an attrition rate of $30 \%$, based on former similar projects). Participants are released for one working hour (T1) and half an hour (T2-T4) to conduct the interview. If the interview is conducted in the workplace, an isolated workstation is accessible to guarantee privacy. The research coordinator schedules telephone appointments and informs respondents that research assistants from graduate programmes in psychology and criminology supervised by a clinical psychologist will conduct the interviews. For participants who are on a sick leave, an additional interview is scheduled at the time of their return to work, if it does not coincide with the time of planned measures. The interviews are recorded by a digital recorder and transcribed later for qualitative analysis purposes. In complement to the interviews with workers exposed to a PTE and in order to meet the fifth objective of this study (ie, perceptions towards the support being offered), key informants of the MYSS-UI (eg, managers, human resources representatives, occupational health and safety representatives, and peer helpers) will be identified with the help of MYSS-UI high management. Sample size will depend on the optimal representation of each stakeholder category (eg, managers, human resources-the total number of interviews is estimated to be about 20). The recruitment procedure for this population will be similar to the one previously mentioned for workers.

\section{Sample size}

Based on power analysis, a total of 222 exposed workers is planned to be recruited: 148 at the MYSS-UI (74 with peer support and 74 without peer support) and 74 at the MYSS. Based on power analysis and after calculating the loss of $30 \%$ of participants over the 12 months of the 
study, 52 subjects per cohort are necessary to conduct this study. In a repeated measures design, this number is sufficient to detect a moderate effect size with three groups with a statistical power of $80 \%$ and a type I error of $5 \% .{ }^{33}$ With an expected moderate effect size and a maximum of 14 predictor variables included in the regressions, the 156 participants are sufficient to achieve a minimum statistical power of. 80 .

\section{Assignment}

Given that peer support programmes are based on a philosophy of inclusion, randomisation may fundamentally alter the peer service under investigation. ${ }^{9}$ For this reason, after being evaluated by our team for their eligibility, participants are either assigned to the first or second cohort depending on whether they chose to receive the peer support intervention in the MYSS-UI. The third cohort includes workers from MYSS who receive standard organisational support without support from peers.

\section{Measures of quantitative data}

Validated self-administrated questionnaires are used at four measurement times (1, 2, 6 and 12 months) to assess psychological distress as well as work functioning. The same questionnaires are used across the four measurement times, excluding acute stress disorder and Perception of the Prevalence of Aggression Scale (POPAS) measured only at T1 and PTSD measured between T2 and T4. Status of variables (exposure, primary outcomes, secondary outcomes, effect modification and potential confounding variables) are described in table 2.

\section{General measures}

Demographic information

Demographic data regarding sex, age, marital status, employment status, employment type, years of experience and duty station are assessed at baseline. The PTE is also measured at baseline with an open question. Information regarding the number of times participants used peer support or any other kind of support (eg, doctor, psychologist, nurse) relatively to the PTE is collected at each measurement time. Information regarding other potential PTE after the one assessed at baseline is also collected between $\mathrm{T} 2$ and $\mathrm{T} 4$.

POPAS. ${ }^{3435}$ This scale was designed to assess experiences of aggressive incidents workers may have encountered in their workplace in the last 12 months. The POPAS contains 16 items representing three forms of violence (eg, verbal threats, severe physical violence, intimidation) ranging from 1 (never) to 5 (very often). The validated French version of the POPAS showed a good to excellent construct validity when using a three-factor structure: 'verbal violence,' 'physical violence' and 'violence against oneself.' 35

\section{Psychological distress}

Acute Stress Disorder Scale (ASDS). ${ }^{36}$ ASDS indexes acute stress disorder using 19 items ranging from 1 (not at all) to 4 (very much), with items taken from the DSM-IV-TR diagnosis of acute stress disorder. Questions cover

Table 2 Type of variables

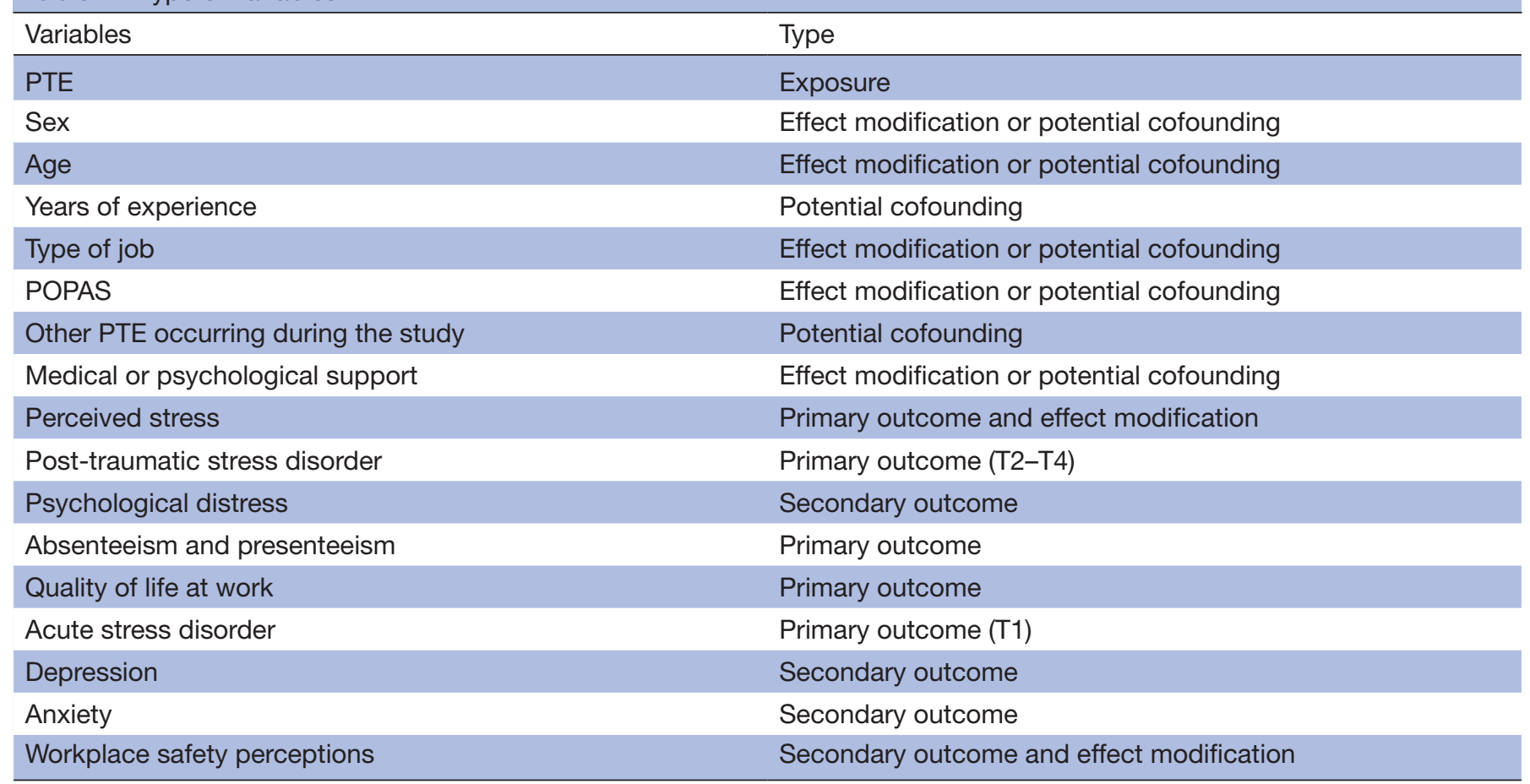

POPAS, Perception of the Prevalence of Aggression Scale; PTE, potentially traumatic events. 
the period of time between the event and the present moment (less than a month). Internal consistency and test-retest $\alpha$ were found to range from good to excellent. ASDS has also shown to have between good and excellent convergent validity with the Acute Stress Disorder Structured Interview. ${ }^{36}$

Post-traumatic Stress Disorder Checklist (PCL5) ${ }^{37}$ The PCL5 assesses the 20 DSM-5 symptoms of PTSD, with 20 items ranging from 0 (not at all) to 4 (extremely), with four clusters covering intrusion symptoms (cluster B), persistent avoidance (cluster $\mathrm{C}$ ), negative alterations in cognitions and mood associated with the traumatic events (cluster D), and hyperarousal (cluster E). Questions cover the last month. PCL5 scores have shown excellent internal consistency, a good internal reliability at test-retest. ${ }^{38} 39$ Full provisional PTSD diagnosis (ie, cut-point at 33) will be calculated, as well as provisional PTSD diagnosis. The latter will be calculated by treating each item rated as at least 'Moderately' as a symptom endorsed, then including at least one symptom in each category from B to E.

Perceived Stress Scale. ${ }^{40}$ This scale was designed to measure the degree to which situations in one's life are appraised as stressful. The scale contains 14 items ranging from 0 (never) to 4 (very often) and covers the last month. Reported Cronbach's $\alpha$ for the French version was good. ${ }^{41}$

Beck Depression Inventory $(B D I) .{ }^{42}$ The BDI assesses the severity of depression in adults and adolescents, with 21 items ranging from 0 to 3 , each level indicating a more severe aspect of the symptom. Two items have $\mathrm{a} / \mathrm{b}$ choices for the same level, representing either more or less of the symptom. BDI scores have shown excellent internal consistency. A moderately high correlation was found to be with the Hamilton Rating Scale for Depression-Revised (HRSD-R) to assess the convergent validity, and a moderate discriminative validity was found, assessed with the Hamilton Anxiety Rating Scale-Revised (HARS-R). ${ }^{43}$

Beck Anxiety Inventory (BAI). ${ }^{44}$ The BAI assesses the severity of anxiety with 21 questions with four choices (from not at all to severely). Questions cover the last week. Psychometric properties have shown an excellent internal consistency and a good internal reliability at test-retest. Convergent validity assessed with the HARS-R was found to be moderate while the correlation with the HRSD-R for assessing divergent validity was found to be weak. ${ }^{44}$

\section{Work functioning}

The Professional Quality of Life Scale (ProQOL) version $5 .{ }^{45}$ The ProQOL measures the professional quality of life using two subscales: 'compassion satisfaction' and 'compassion fatigue.' The latter is subdivided into 'burnout' and 'secondary trauma,' with 30 items ranging from 1 (never) to 5 (very often). Questions cover the last 30 days. The $\alpha$ reliabilities for the scale were found to be between acceptable (burnout) and good (compassion fatigue and compassion satisfaction) by Stamm. ${ }^{45}$

Presenteeism and absenteeism (StatCan). This scale measures absenteeism and productivity at work based on nine questions: for two of them, participants answer with a number, two questions with a Likert scale ranging from 1 (all the time) to 6 (no answer), and five questions with a Likert scale ranging from 1 (all the time) to 5 (never). It has been created by Statistics Canada, a federal organisation whose purpose is to provide statistical information and analysis about Canada's economic and social structure.

Work Safety Scale (WSS). ${ }^{46}$ The WSS measures the perception of workplace security for employees, with 50 items. There are five subsections: job safety, coworker safety, supervisor safety, management safety practices and safety programmes (policies). For each section, there are 10 choices. Cronbach's $\alpha$ was found to be good by Hayes $e t$ $a l^{46}$

\section{Measures of qualitative data}

A semistructured grid was designed to conduct interviews with workers. Themes used are in connection with the PTE, namely nature of the incident, nature of support offered and provided by different sources, feelings right after the incident (ie, behaviours, reactions, state of mind), report to the employer as well as worker's needs post-PTE, and their perceptions of why the support received was useful or not. During the first interview, the main aim is to understand the process underlying the decision to ask for or accept organisational support and the reasons motivating this decision. Examples of questions asked include: 'Please tell me what happened immediately after the event?' /'How were you feeling then?'/'Who did you talk to after?'. For each question, subquestions and elements to obtain are indicated for the interviewer. For instance, for the question 'Please tell me what happened immediately after the event?' the following instructions were given to the interviewer:

1. Elements sought: what the participant remembers after the event, what support was offered and received and how it was perceived by the participant.

2. Possible prompts: Who did you speak to? How did they react? How did the conversation go? What was the most helpful aspect of this interaction? What was your impression of their reaction/support? Who did you most want to confide to? If you had to describe the support you received from your supervisor/ organisation/colleagues/peer support programme, what words would you use? In an ideal world, what could your supervisor/organisation/colleagues/peer support programme have done to support you better?

For the following three interviews, themes used are in connection with the nature of support offered and provided by different sources since the PTE, the evolution of their feelings regarding the incident (ie, behaviours, reactions, states of mind) as well as the organisational response to their needs.

A semistructured grid was also built to conduct the interviews with key informants. Themes used are in connection with their perceptions towards the support provided to PTE victims and on the PTIT. For example, 


\begin{tabular}{ll}
\hline Table 3 Potential bias & \\
\hline Domain & Propositions \\
\hline $\begin{array}{l}\text { Random sequence } \\
\text { generation }\end{array}$ & $\begin{array}{l}\text { The nature of the study does not allow for randomisation. Participants of the MYSS-UI may have } \\
\text { chosen to receive the peer support or not based on prior victimisation or the type of PTE. We will } \\
\text { control for these variables, among others, when proceeding to analyses. }\end{array}$ \\
Blinding of participants & $\begin{array}{l}\text { All participants are informed that the purpose of the study is to assess the support offered to } \\
\text { workers experiencing a PTE at work in two organisations. Participants of MYSS-Ul are informed } \\
\text { of this aim after choosing to receive or not peer support. They are not explicitly told that cohorts } \\
\text { receive different protocols and that they will be compared. We made this choice in order to reduce } \\
\text { performance bias among workers. }\end{array}$ \\
\hline Incomplete outcome data & $\begin{array}{l}\text { For each main outcome, we will describe the completeness of outcome data, including attrition and } \\
\text { exclusions from the analysis. If less than 5\% of the participants refuse follow-up, we will censor the } \\
\text { data at the point of loss of contact. Otherwise, we will adjust the model using sociodemographic } \\
\text { information at time 1 and maximum likelihood estimations instead of listwise deletion. Moreover, we } \\
\text { will try to document the reasons for discontinuation in contacting participants once by telephone } \\
\text { and once by email (if necessary). } \\
\text { We will provide analysis for all the outcomes of the present protocol, which will avoid reporting bias. } \\
\text { A non-significant result is a result itself and should be known by other researchers. }\end{array}$ \\
\hline Selective reporting &
\end{tabular}

MYSS-UI, Montreal Youth Social Services-University Institute; PTE, potentially traumatic events.

key informants will be asked to discuss about the perceived effectiveness of the PTIT, its impact on the organisational culture and their perceptions of the organisational culture towards PTE.

\section{Potential sources of bias}

In accordance with Cochrane recommendations as regards risk of bias, we considered and will consider potential sources of bias (see table 3).

\section{Proposed analyses}

\section{Quantitative analysis}

Quantitative analysis will be processed by the IBM SPSS V.21 software. Before analysis, the data will be studied to determine their compliance with the homoscedasticity hypothesis (Cochran test) and normality. ${ }^{47}$ Variables will be transformed when necessary. The adopted significance level will be set at 0.05 and bilateral tests will be used. The variable distributions will be described in terms of averages or percentages with appropriate CIs. In all analyses, we will control the potentially confounding covariates such as sex, age, years of experience, type of PTE and work leave experienced. Analyses of covariance will be carried out if there are differences between groups. In addition, moderation analysis will be conducted to assess whether potential effects related to sex are associated with gender roles or with biological sex.

The effects of each intervention will be first analysed using repeated measures analysis of variance on variables with a continuous score to confirm the presence or absence of improvements within each group (multivariate analyses of covariance). $\chi^{2}$ tests will be used to compare the three cohorts in terms of the presence/absence of an ASD (using ASDS) and PTSD (using the PCL) to the ratings of 2, 6 and 12 months.

\section{Qualitative analysis}

Qualitative analysis will be performed with the software package QSR-NVivo V.11. We will conduct a thematic analysis that consists of (1) reading transcripts in order to get a general sense, (2) dividing the interviews into units of meaning, (3) identifying and interpreting of the themes, (4) summarising themes for each participant and, lastly, (5) presenting a general portrait of the themes and subthemes of each participant. Two research-trained organisational psychologists will code the interviews and the final analysis will be conducted by a researcher specialised in qualitative analysis. They will look for specific information on the perceived needs and perceived usefulness of the intervention.

\section{Ethics, benefit and dissemination}

The project has ethical approval (Ref. MP-CJM-IU-15-02) of MYSS-UI and MYSS. Consents must be signed and returned to the team of researchers before the inclusion of any participant. Participants can stop being part of the study at any moment. To preserve anonymity, we pseudonymise ID information, with the attribution of a number to each participant. Data that identify participants will be kept in password-protected electronic storage with the option of encryption or in locked cabinets in the main researcher's office for self-administered paper questionnaires. When collecting data by questionnaire and interview with exposed workers, some participants may experience psychological discomfort (eg, anxiety) due to recalling certain information related to the traumatic event experienced. The interviewers are trained to be able to detect this type of participants and to provide help resource references.

Expected benefits from this research relate to modifications of the health and safety programmes of the two organisations (MYSS-UI and MYSS), and other youth 
social services in Canada and worldwide. Recommendations will also be offered to all organisations on staff support (eg, motives for using it or not, needs, satisfaction).

Our dissemination plans consist of local meetings with every unit. We will report our findings back to the management of MYSS-UI and MYSS, with inclusion of Health and Safety Office representatives at face-to-face meetings. We will submit presentations to national and regional conferences and organisations aimed at health and social services. We will write a report for the workers and for the institute that funded the project (Institut de recherche Robert-Sauvé en santé et en Sécurité du travail). The Institute has no authority over the collection, management, analysis and interpretation of data. We will also submit papers to peer-reviewed journals and to academic and scientific conferences, prioritising open-access publication.

The authors have no conflicts of interest to disclose. There were no sources of funding that could have influenced the outcome of this work.

\section{DISCUSSION}

This study will provide evidence on whether using a peer support intervention affects positively psychological distress and work functioning for workers experiencing PTE. This study will also allow for a better understanding of the needs of workers with regard to support. This research study will have an impact on the health and safety programmes of both organisations, regardless of the direction of the results, as both are open to changing their politics for the best interest of their workers.

\section{CONCLUSION}

This study seeks to investigate if a peer support programme is an effective method to prevent impairments in wellbeing and work functioning. This has implications for all youth social services.

Correction notice This paper has been amended since it was published Online First. Owing to a scripting error, some of the publisher names in the references were replaced with 'BMJ Publishing Group'. This only affected the full text version, not the PDF. We have since corrected these errors and the correct publishers have been inserted into the references.

Acknowledgements The authors wish to thank Josette Sader and Josianne Lamothe for their proofing.

Contributors Given the identical initials between Stephane Guay and Steve Geoffrion, the latter will be referred to as SGe.Contributors SG and HB conceived the study design. SG, HB, SGe and NT were involved in the grant funding writing, trial design and ethics approbation. JG and SGe were involved in ethics approbation, manuscript preparation and statistical analysis. SG, NT and SGe are involved in monthly meetings with the organisations to assess the intervention development and thus until the end of the recruitment. SG and SGe are in charge of knowledge transfer. All the authors provided a critical revision and gave approval for the manuscript to be submitted.

Funding The writing of this paper was supported by funding grant from the Institut de recherche Robert-Sauvé en santé et en Sécurité du travail.

Competing interests None declared.
Ethics approval Research Ethics Board of Montreal Youth Social Services University Institute.

Provenance and peer review Not commissioned; externally peer reviewed.

Open Access This is an Open Access article distributed in accordance with the Creative Commons Attribution Non Commercial (CC BY-NC 4.0) license, which permits others to distribute, remix, adapt, build upon this work non-commercially, and license their derivative works on different terms, provided the original work is properly cited and the use is non-commercial. See: http://creativecommons.org/ licenses/by-nc/4.0/

(c) Article author(s) (or their employer(s) unless otherwise stated in the text of the article) 2017. All rights reserved. No commercial use is permitted unless otherwise expressly granted.

\section{REFERENCES}

1. Tolin DF, Foa EB. Sex differences in trauma and posttraumatic stress disorder: a quantitative review of 25 years of research. Psychol Bull 2006;132:959-92.

2. Lanctôt N, Guay S. The aftermath of workplace violence among healthcare workers: a systematic literature review of the consequences. Aggress Violent Behav 2014;19:492-501.

3. Diagnostic and statistical manual of mental disorders: $d s m-5^{\mathrm{TM}}$. 5th ed. VA: ArlingtonAmerican Psychiatric Publishing;, 2013.

4. Dameron-Brown RM. Examining the lived experiences of Child Welfare Workers. Minnesota: Walden University, 2015.

5. Enosh G, Tzafrir SS, Stolovy T. The development of Client Violence Questionnaire (CVQ). J Mix Methods Res 2015;9:273-90.

6. Littlechild B, Hunt S, Goddard C, et al. The effects of violence and Aggression from parents on Child Protection Workers' Personal, Family, and Professional Lives. Sage Open 2016;6:215824401562495-12.

7. Harris B, Leather P. Levels and consequences of exposure to Service user violence: evidence from a sample of UK Social Care Staff. Br J Soc Work 2012;42:851-69.

8. Winstanley S, Hales L. Prevalence of Aggression towards Residential Social Workers: do qualifications and experience make a difference? Child Youth Care Forum 2008;37:103-10.

9. Creamer MC, Varker T, Bisson J, et al. Guidelines for peer support in high-risk organizations: an international consensus study using the Delphi method. J Trauma Stress 2012;25:134-41.

10. Everly Jr GS, Mitchell JT. Critical incident stress management (CISM): Coping with the aftermath of violence. Ellicott City, MD: Chevron Publishing, 1999.

11. Mitchell JT, Everly GS. Critical incident stress debriefing (CISD) and the prevention of work-related traumatic stress among high risk occupational groups. In: Dand G, Everly S, Lating JM, eds. Psychotraumatology: key papers and core concepts in post-traumatic stess. Plenum series on stress and coping. New York: plenum Press, 1995.

12. Resnick SG, Rosenheck RA. Integrating peer-provided services: a quasi-experimental study of recovery orientation, confidence, and empowerment. Psychiatr Serv 2008;59:1307-14.

13. Flannery RB, Juliano J, Cronin S, et al. Characteristics of assaultive psychiatric patients: fifteen-year analysis of the Assaulted Staff Action Program (ASAP). Psychiatr Q 2006;77:239-49.

14. Flannery RB. Staff victims of elder patient abuse and the Assaulted Staff Action Program (ASAP): preliminary empirical inquiry. Am J Alzheimers Dis Other Demen 2003;18:93-6.

15. Flannery RB, Anderson E, Marks L, et al. The Assaulted Staff Action Program (ASAP) and declines in rates of assault: mixed replicated findings. Psychiatr Q 2000;71:165-75.

16. Vogt J, Leonhardt J, Köper B, et al. Economic evaluation of CISM--a pilot study. Int J Emerg Ment Health 2004;6:185-96.

17. Freeman DG, Carson M. Developing workplace resilience: the role of the peer referral agent diffuser. Journal of Workplace Behavioral Health 2007;22:113-21.

18. Gould M, Greenberg N, Hetherton J, Stigma HJ. Stigma and the military: evaluation of a PTSD psychoeducational program. J Trauma Stress 2007;20:505-15.

19. Guay S, deTournay-Jetté E, Beaulieu-Prevost D, et al. Earlyinterventions designed to help victims of a violent crime: a systematic literature review of psychological outcomes. Bologna, Italy: 13thCongress of the European Society for Traumatic Stress Studies, 2013.

20. Horejsi C, Garthwait C, Rolando J. A survey of threats and violence directed against child protection workers in a rural state. Child Welfare 1994;73:173-9. 
21. Ringstad R. CPS: client violence and client victims. Child Welfare 2009;88:127-44.

22. Deutsch SA, Lynch A, Zlotnik S, et al. Mental Health, Behavioral and developmental issues for Youth in Foster Care. Curr Probl Pediatr Adolesc Health Care 2015;45:292-7.

23. Woods SB, Farineau HM, McWey LM. Physical health, mental health, and behaviour problems among early adolescents in foster care. Child Care Health Dev 2013;39:220-7.

24. Violence $\mathrm{H}$. And aggression: short-term management in mental health, health and community settings. NICE Guideline NG 2015.

25. Geoffrion S, Ouellet F. When rehabilitation hurts? physical violence directed against behaviour technicians in juvenile rehabilitation centres. Criminologie 2013;46:263-89.

26. Geoffrion S. Aggression and accountability: how caregivers and law enforcers cope. Montreal: Université de Montréal. https://papyrus. bib.umontreal.ca/xmlui/handle/1866/12327

27. Horwitz MJ. Work-Related trauma effects in Child Protection Social Workers. J Soc Serv Res 2006;32:1-18.

28. Perron BE, Hiltz BS. Burnout and secondary trauma among forensic interviewers of abused children. Child and Adolescent Social Work Journal 2006;23:216-34.

29. Regehr C, Hemsworth D, Leslie B, et al. Predictors of post-traumatic distress in child welfare workers: a linear structural equation model. Child Youth Serv Rev 2004;26:331-46.

30. Rhee YS, Ko YB, Han IY. Posttraumatic growth and related factors of child protective service workers. Ann Occup Environ Med 2013;25:6-10.

31. Bilodeau H, Marchand A, Berthelette D, et al. Analyse d'un protocole d'intervention post-traumatique et de mesures de gestion associées au Centre jeunesse de Montréal-Institut universitaire. Report \# R-819). Montréal, Québec: IRSST 2014.

32. Bryant RA. Early intervention for post-traumatic stress disorder. Early Interv Psychiatry 2007;1:19-26.

33. Faul F, Erdfelder E. GPOWER: a priori, post-hoc, and compromise power analyses for MS-DOS Bonn, Germany: Bonn University, 1992.
34. Oud N. The perception of prevalence of Aggression Scale (POPAS) Questionnaire. Amsterdam: Connecting, 2001.

35. Geoffrion $S$, Giguère $C$, Fortin $M$, et al. Validation of the french canadian version of the perception of prevalence of aggression scale (POPAS) with a sample of youth workers. Revue de Psychoéducation In press.

36. Bryant RA, Moulds ML, Guthrie RM. Acute stress disorder scale: a self-report measure of acute stress disorder. Psychol Assess 2000;12:61-8.

37. Weathers F, Litz B, Keane T, et al . The PTSD checklist for DSM-5 (PCL-5) http://wwwptsdvagov . 2014.

38. Blevins CA, Weathers FW, Davis MT, et al. The posttraumatic stress disorder Checklist for DSM-5 (PCL-5): Development and Initial Psychometric Evaluation. J Trauma Stress 2015;28:489-98.

39. Wortmann JH, Jordan AH, Weathers FW, et al. Psychometric analysis of the PTSD Checklist-5 (PCL-5) among treatment-seeking military service members. Psychol Assess 2016;28:1392-403.

40. Cohen S, Kamarck T, Mermelstein R. A global measure of perceived stress. J Health Soc Behav 1983;24:385-96.

41. Lesage F-X, Berjot S, Deschamps F. Psychometric properties of the french versions of the perceived stress Scale. Int J Occup Med Environ Health 2012;25:178-84.

42. Beck AT, Steer RA, Brown GK. Manual for the beck depression inventory-II. San Antonio, TX: Psychological Corporation, 1996.

43. Dozois DJA, Dobson KS, Ahnberg JL. A psychometric evaluation of the Beck Depression Inventory-II. Psychol Assess 1998;10:83-9.

44. Beck AT, Epstein N, Brown G, et al. An inventory for measuring clinical anxiety: psychometric properties. J Consult Clin Psychol 1988;56:893-7.

45. Stamm BH. The ProQOL manual. the Professional Quality of Life Scale: compassions satisfaction, burnout \& compassion fatiguel secondary trauma scales. Baltimore, MD: Sidran Press, 2005.

46. Hayes BE, Perander J, Smecko T, et al. Measuring perceptions of workplace safety: development and validation of the work safety scale. J Safety res 1998;29:145-61.

47. Tabachnick BG, Fidell LS, Osterlind SJ, et al; Los Angeles. 5th ed. California: Pearson, 2001. 